\title{
The role of mathematical models and scoring systems in detecting dysgerminoma in a young patient
}

\author{
Nabil Abdalla, Joanna Winiarek, Agnieszka Timorek, Wlodzimierz Sawicki, \\ Krzysztof Cendrowski
}

Department and Clinic of Obstetrics, Gynecology and Oncology, Second Faculty of Medicine, Medical University of Warsaw, Poland

A 20-year-old Caucasian woman presented at the emergency unit with a complaint of lower abdominal pain, which was had been present for a few months but worsened on that day. The patient had no family history of cancer. She denied weight loss, vomiting, diarrhea, and constipation.

The patient is a virgin, with irregular and heavy menses (6-7 days, every 33-45 days). Transabdominal ultrasound was performed by an inexperienced examiner using Philips iu $22 z$ apparatus. Gray-scale ultrasound examination revealed an irregular heteroechogenic solid mass $(114 \times 77 \times 72 \mathrm{~mm}$ in size) in the pelvis, protruding into the abdomen on the left side (Fig. 1). There were no acoustic shadows and the patient reported no pain during the examination. There was only a small amount of fluid below the mass (Fig. 2). Color Doppler examination of the mass revealed moderate vascularization, assessed as score 3 according to the terms and definitions established by the International Ovarian Tumor Analysis (IOTA) group (Fig. 3). The second ovary was irregular $(40 \times 28 \mathrm{~mm})$, in close proximity to the pelvic mass with hypoechogenic areas, suggestive of metastases. The examination revealed a hypoechogenic area of $15.5 \times 17 \mathrm{~mm}$ in the pancreas, suggestive of metastases (Fig. 4). The liver and the kidneys had no focal lesions. The mass was considered as malignant according to the subjective assessment by the inexperienced ultrasound examiner.

Ten selected mathematical models and scoring systems, based on clinical features, ultrasound examination and serum concentration of tumor markers, were used to assess the mass by the inexperienced examiner. The risk of Malignancy Algorithm (ROMA) revealed a high (10.24\%) risk for ovarian cancer malignancy. Malignant criterion as irregular solid mass in the absence of other benign criteria suggested a malignant nature of the mass, according to the simple rules of the IOTA group. The results of the IOTA logistic regression models LR1 and LR2 were $43.7 \%$ and $23.5 \%$ respectively, indicating a malignant character of the lesion. The pelvic mass was considered to be malignant according to the risk of malignancy indices RMI I-IV, whose results were $512,683,512$ and 1366 , respectively. The Gynecologic Imaging Report and Data System classified the mass as grade 4, i.e. "probably malignant". The ADNEX model revealed a $68.2 \%$ risk of malignancy, with $27.4 \%$ risk of being stage II-IV ovarian cancer. After obtaining the patient's consent, fertility-sparing surgical operation was performed. The final diagnosis was dysgerminoma, stage IV according to the International Federation of Gynecology and Obstetrics (FIGO) criteria. In the case of our patient, advanced stage of the ovarian cancer might have facilitated the diagnosis as the changes were evident. On the other hand, the rarity of the disease, young age of the patient, and non-specific symptoms may cause diagnostic difficulties. Systemic approach, with the use of clinical, ultrasound and tumor markers, may help not to overlook ovarian cancer even in younger patients when assessed by inexperienced examiners.

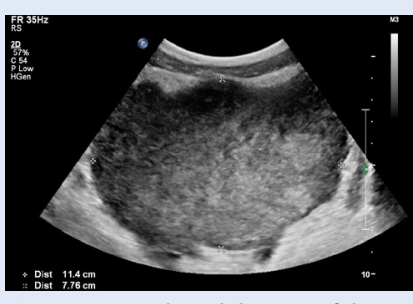

Figure 1. Irregular solid tumor of the left ovary

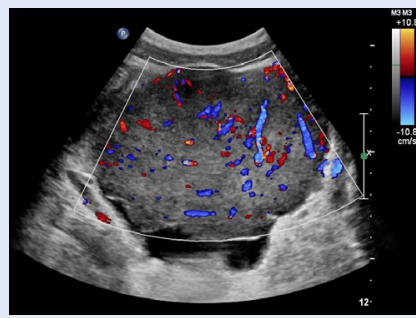

Figure 3. Moderate vascularization of the tumor

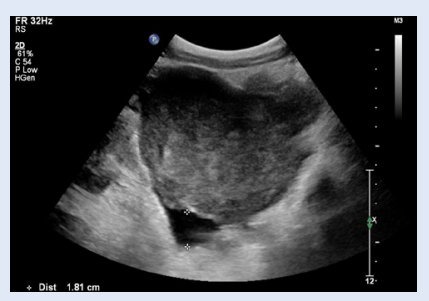

Figure 2. Small amount of fluid beneath the tumor

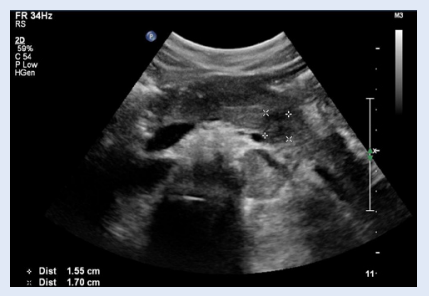

Figure 4. Pancreatic metastases

Corresponding author:

Nabil Abdalla

Department and Clinic of Obstetrics, Gynecology and Oncology, Second Faculty of Medicine, Medical University of Warsaw, Kondratowicza St. 8, 83-242 Warsaw, Poland tel.:/fax: +48223265380

e-mail:drnabilabdalla@yahoo.com 